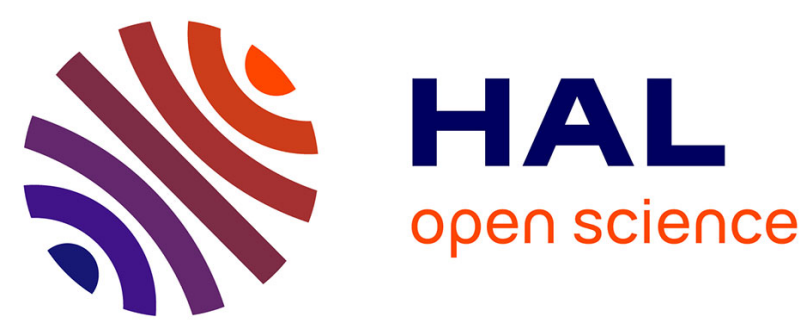

\title{
Network Architecture and Essential Features for 5G: The SESAME Project Approach
}

\author{
Leonardo Goratti, Cristina E. Costa, Jordi Perez-Romano, Oriol Sallent,
} Cristina Ruiz, August Betzler, Pouria Sayyad Khodashenas, Seiamak Vahid, Karim M. Nasr, Babangida Abubakar, et al.

\section{To cite this version:}

Leonardo Goratti, Cristina E. Costa, Jordi Perez-Romano, Oriol Sallent, Cristina Ruiz, et al.. Network Architecture and Essential Features for 5G: The SESAME Project Approach. 12th IFIP International Conference on Artificial Intelligence Applications and Innovations (AIAI), Sep 2016, Thessaloniki, Greece. pp.676-685, 10.1007/978-3-319-44944-9_60 . hal-01557604

\section{HAL Id: hal-01557604 https://hal.inria.fr/hal-01557604}

Submitted on 6 Jul 2017

HAL is a multi-disciplinary open access archive for the deposit and dissemination of scientific research documents, whether they are published or not. The documents may come from teaching and research institutions in France or abroad, or from public or private research centers.
L'archive ouverte pluridisciplinaire HAL, est destinée au dépôt et à la diffusion de documents scientifiques de niveau recherche, publiés ou non, émanant des établissements d'enseignement et de recherche français ou étrangers, des laboratoires publics ou privés.

\section{(c)(1)}

Distributed under a Creative Commons Attribution| 4.0 International License 


\title{
Network Architecture and Essential Features for 5G: The SESAME Project Approach
}

\author{
L. Goratti , C. E. Costa , J. Perez-Romano ${ }^{2}$, O. Sallent ${ }^{2}$, C. Ruiz ${ }^{3}$, A. Betzler, P. S. \\ Khodashenas, $^{3}$ S. Vahid, K. M. Nasr, B. Abubakar, A. Whitehead, M. Belesioti', \\ and, I. Chochliouros ${ }^{7}$ \\ 'CREATE-NET, Via alla Cascata 56/D, Italy \\ ${ }^{2}$ Universitat Politecnica de Catalunya (UPC), Spain, ${ }^{3}$ i2CAT, Spain \\ ${ }^{4}$ University of Surrey, UK, 'University of Brighton, UK, \\ ${ }^{6}$ ip.access, UK, 'Hellenic Telecommunications Organization, Greece \\ Contact Email: leonardo.goratti@create-net.org
}

\begin{abstract}
The outstanding and continuous growth of the request of mobile broadband Internet access is creating the unprecedented need to rethink most of the design paradigms of the mobile network. Such trend is accompanied by remarkable progresses of miniaturised electronics, together with the proliferation of social services and computation intensive applications such as high definition video. On one hand, the current mobile network is unable to deliver sufficiently high data rates per user in order to support this growth, and a possible solution is provided by the dense deployment of small cell devices. On the other, mobile operators are struggling to lower costs of deployment and maintenance while keeping profitable revenues. This paper aims to provide overview of the solution developed by the 5G-PPP SESAME project. SESAME proposes to leverage on the concept of Small Cell-as-a-service (SCaaS), providing the complete architectural solution to deploy cloud-enabled small cells. The key innovations developed by SESAME include the deployment of computation capabilities at the mobile network edge, and to exploit virtualisation techniques to manage and orchestrate dense small cell scenarios and different use cases.
\end{abstract}

Keywords: 5G, scaas, mobile edge computing, self-organising networks, small cell virtualisation.

\section{Introduction}

In recent years the way mobile users access and consume contents has dramatically changed due to remarkable progresses of miniaturised electronics and the proliferation of portable and user friendly devices including smartphones and tablets [1]. As shown in [2], during the third quarter of 2015, almost 3.4 billion subscriptions were registered worldwide. At the same time new social services and applications 
have become so popular that mobile connectivity is a preferred way for users. It is evident that with the current pace of growth even LTE-Advanced (LTE-A) will fail to support such high traffic.

Recently the 5G Infrastructure Public Private Partnership (5G-PPP) [3] has started its activities to create the next generation of mobile network. The SESAME project [4] is one amid the nineteen projects which were recently funded. As mentioned above, the emergence of innovative services and increased network availability motivate and drive further mobile user's engagement, creating a loop of increasing expectations and demand [5]. It is worth registering that mobile broadband penetration has indeed risen to $85.5 \%$ in the OECD area, meaning more than four wireless subscriptions for every five inhabitants, while the penetration rate in June 2014 was just 76\% [6]. To cope with this large growth, METIS project [7] has defined a large number of use cases that $5 \mathrm{G}$ technology shall serve with superior performance over previous generations of cellular technology.

To meet growing users' demand the solution is offered by denser networks in which small form factor small cell (SC) devices can be deployed in large amounts and operate over licensed spectrum. This trend is also regarded to as network densification. Several approaches and technologies are currently converging in the new 5G mobile network as generally discussed in [8]. In particular, besides new physical layer solutions, Software-Defined Networking (SDN) [9], and Network Functions Virtualisation (NFV) $[10,11]$ are making their way toward a programmable network solution. Borrowing from cloud computing concepts such as Infrastructureas-a-Service (IaaS) and more recently XaaS (anything-as-a-Service) have been adopted also in the mobile network domain. These approaches facilitate the decoupling between service provider, infrastructure provider and network provider. As a consequence, besides traditional Mobile Network Operators (MNO), new types of Virtual Network Operators (MVNO) and Over-The-Top (OTT) service providers can find unprecedented opportunities in $5 \mathrm{G}$.

The SESAME project develops the concept of Small Cell-as-a-Service (SCaaS), which leverages on the separation between traditional market roles, with the aim to make resources available through network virtualisation. In this context, SESAME will maximise the opportunities offered by opening small cells to multi-tenancy. This latter concept, in opposition to typical mobile operators which deploy their own network infrastructure in competition with others, encourages both traditional and new market entrants to share the infrastructure. In this case operators can differentiate based on their service offers rather than on network connectivity. To manage the dense SC network, including mitigating interference and assign resources dynamically, the ETSI NFV Industry Specification Group (ISG) has developed the Management and Orchestration (MANO) framework that constitutes a solution for managing virtualised small cells [12]. This flexible and dynamic system allows operators to reduce CAPEX and OPEX as required in the next generation of mobile networks. 


\subsection{SESAME Enabling Technologies}

Small Cells. Small Cells Small cells have become pivotal in today's 4G access. Small cells installation is an effective way to achieve greater performance and capacity to both indoor and outdoor places: they provide improved cellular coverage, higher capacity and applications for homes and enterprises, as well as in dense metropolitan and rural areas [13]. Their role is crucial for providing services in specific high traffic places such as office areas, dense urban areas, stadiums, shopping malls, concert venues, and generally, places with (tactic or sporadic) high end-users density [14].

Mobile-Edge Computing. ETSI Mobile-Edge Computing (MEC) offers computing capabilities at the network edge and bring different services near to the mobile subscribers. Providing edge cloud capabilities allows to enable accelerated services, content and application thanks to increased network responsiveness. The approach proposed is to deploy a MEC server between the mobile core and the Radio Access Network (RAN). Typical services which can benefit from mobile-edge computing include Internet-of-Things, augmented reality and data caching.

Virtualisation. Virtualisation of the communication infrastructure, such as core/edge network elements and access points/macrocells, has been extensively studied by several industry and research initiatives up to now. Recently, its applicability to the small cell infrastructure has now started to receive increasing attention. The remainder of this paper is organised as follows. In Section 2 we describe the general principles behind the SESAME project. In Section 3 we provide detailed description of the SESAME system and conclusions are drawn in Section 5.

\section{SESAME: Small Cell Coordination for Multi-Tenancy and Edge Services}

The SESAME project targets innovations around the placement of network intelligence and services in the network edge through NFV and cloud computing. Through the evolution of the SC concept, already mainstream in 4G, SESAME expects to exploit its full potential in challenging highly dense $5 \mathrm{G}$ scenarios. SESAME targets providing SCaaS and to consolidate multi-tenancy in communication infrastructures, allowing several operators/service providers to engage in new sharing models, obtaining higher capacity on the access side and exploiting edge computing capabilities.

The key innovations proposed by SESAME focus on the novel concepts of virtualising SC networks by substantially evolving the SC concept under the paradigms of a multi-operator (i.e. multi-tenancy) enabling framework and an edgebased, virtualised execution environment. SESAME leverages on the capability to deliver intelligence directly to the network's edge, in the form of virtual network appliances. The provisioning of multi-operator SC networks is optimized for the most promising scenarios and use cases. 
SESAME develops and will demonstrate an innovative architecture, capable of providing SC networks to multiple operators. SESAME fosters the concept of logical partitioning of the SC network in multiple isolated slices, virtualising and partitioning small cells capacity to multiple tenants. SESAME supports enhanced multi-tenant edge cloud services combining SCs with micro-server facilities. The unique characteristics of the SESAME approach allows new SC operators (real estate companies, municipalities, etc) to enter the value chain deploying access infrastructure in specific high traffic demanding areas, and acting as neutral host providers, offering to existing mobile operators on-demand access to network resources.

\subsection{SESAME Design Principles}

The SESAME architecture was driven by design principles extracted from the identification and analysis of high-impact use cases and stakeholders' requirements. Each stakeholder involved in the process behaves according to different objectives. MNOs can achieve lower total cost of ownership combining own resources with outsourced access capacity and computing; VMNOs benefit of wireless services without owing the physical infrastructure; Venue Owners as new market entrants can benefit from becoming local network operators; End Users can enjoy personalised services with superior quality of experience.

In order to maximise user experience, the architecture should take into account various user requirements such as per-user data rate and latency, robustness and resiliency, mobility, seamless user experience and context-awareness. On the other hand, network requirements must be met in order to allow efficient operation and management. The latter include scalability, network capacity, automated system management and configuration, advanced Self-Organizing Network (SON) features, network flexibility, improved coverage, security and flexible spectrum management.

The set of design principles that drives the SESAME architecture is described below. These are derived from both the analysis of use cases and underlying requirements, as well as considering the state-of-the-art in the technological fields relevant to SESAME (e.g. SCs, NFV, cloud computing), and the most prominent standardisation trends. Principle 1: The SESAME system is sustainable and reconfigurable; Principle 2: SESAME offers an infrastructure shared between operators, transparent and neutral; Principle 3: SESAME accelerates the creation of innovative services with superior quality of experience through mobile edge computing; Principle 4: SESAME develops a system which is capable of optimising the usage of radio, storage and computing resources. 


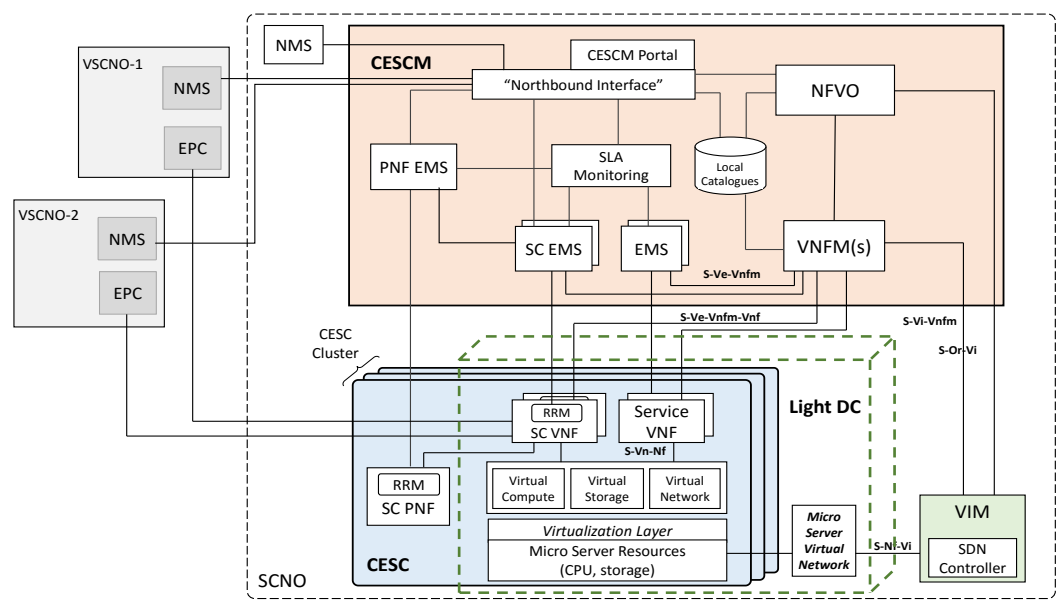

Fig. 1. SESAME System Architecture.

\section{The SESAME System}

SESAME proposes a novel $5 \mathrm{G}$ platform based on small cells, featuring multi-tenancy and edge cloud capabilities, offered to both network operators and mobile users. In the SESAME system shown in Fig. 1, one key design principle is to support the innovative concept of Virtual Small Cell Network Operators (VSCNO). In particular, VSCNOs use the infrastructure deployed by a SCNO.

The SESAME approach enhances the SC with micro-servers, which together form the Light DC execution environment. Micro-servers in the Light DC are connected whereby a dedicated internal network.

\subsection{High-Level Architecture}

The SESAME system in Fig. 1 bases its architecture on the concept of the CloudEnabled Small Cell (CESC), a new multi-operator enabled SC that integrates a virtualised execution platform (i.e. the Light DC) for deploying VNFs, supporting automated network management and executing novel applications and services inside the access network infrastructure. The Light DC features low-power processors and hardware accelerators for time critical operations and provides a highly manageable clustered edge computing infrastructure. SESAMEs Light DC is based on low-power 64-bit processors supported by hardware accelerators (e.g. GPU, DSPs and FPGAs). In addition to the internal network connecting the micro-servers, a suitable backhaul connection provides connectivity for the SESAME system to external packet data networks.

The CESC Manager (CESCM) provides optimized management of the CESC deployment and is a key element of the SESAME architecture. It implements orchestration, NFV management, virtualisation of management views per tenant, Self- 
$\mathrm{x}$ features and radio access management techniques. The Portal is used by externals to request resources or apply (re)configuration of parameters. The NorthBound Interface (NBI) is the connecting point between orchestration, Service Level Agreements (SLAs) and VSCNOs. The SLA Monitoring module provides inputs to both VSCNOs and infrastructure providers about correct execution of the environment, and it enables the orchestration subsystem to react accordingly to possible changes in the network.

Small cells connect to the operator's domain and in particular to the Evolved Packet Core (EPC) and the Network Management System (NMS). The NMS is responsible for communicating with the typical Element Management System (EMS) in the small cells domain, in charge of Fault, Configuration, Accounting, Performance and Security (FCAPS) for physical and virtual functions, as will be clarified shortly. As shown in Fig. 1, SDN is used to configure the forwarding behaviour of traffic inside the Light DC and the chain of VNFs.

\subsection{Essential Features}

The essential innovations brought by the SESAME architecture (Fig.1) delve into virtualising small cells, as well as leveraging on the paradigm of multi-tenancy (i.e. multi-operator) coupled with a virtualised computation environment at the mobile network edge.Multiple network operators will be able to use the SESAME system, each one through its own slice of network resources. The idea is to deploy small cells in which functions are virtualised, and each small cell is also connected to a microserver through appropriate fronthaul technology. A micro-server is based on a nonx86 architecture using 64-bit ARMv8 technology. As anticipated, micro-servers together with the SCs form the CESC, and a number of CESCs form the CESC cluster, which can be shared by multiple operators within a geographical area. SESAME will develop important advances also in network management developing a novel edge-computing architecture and deploying Self-x procedures directly to the network edge. To that end, SESAME will support multi-tenancy through MultiOperator Core Network (MOCN).

\subsection{Functional Split in Small Cells}

SESAME fully embraces network virtualisation, which allows small cell functions to be decoupled from the physical hardware. Indeed, SC functions can be split between Physical Network Function (SC PNF) and virtual (SC VNF), with one SC VNF which can be connected to multiple SC PNF through the fronthaul. Several studies are currently carried forward by the Small Cell Forum to evaluate the implications of different functional splits between physical and virtual [15]. The fact that one SC VNF can connect to several SC PNF offers several advantages: 1) improved coordination of the radio functions (coordinated scheduling, inter-cell interference coordination, etc), 2) enhanced scalability of small cell deployments with simplified management, 3) accelerated life-cycle upgrade enabling new features and 4) flexibility to optimally make the workload placement. 


\subsection{Cloud-Enabled SESAME Environment}

SESAME relies on SDN and NFV to use general-purpose computing and storage hardware at the mobile network edge. Along this direction a micro-scale virtualised execution environment proposed by SESAME consists in the Light DC, which offers the cloud execution environment of SESAME. The Light DC is designed to build a clustered infrastructure with high manageability and optimised to reduce power consumption, cabling, space and costs. This execution platform is used to support the required VNFs that implement the different SC features and the cognitive Self-x management operations. In addition, SESAME will develop several service VNFs, which include virtual video transcoding, virtual caching and virtual load balancer, just to name a few. Both SC and service VNFs shall be described by appropriate descriptor files using either TOSCA, JSON or YAML files.

As shown in Fig. 1, the CESCM includes the SESAME MANO amongst other components. The MANO include the NFV Orchestrator (NFVO), the VNF Manager (VNFM) and the Virtualised Infrastructure Manager (VIM) [12]. The SESAME NFVO provides the automated execution environment for VNFs and Network Services (NSs). The NFVO can deploy a new VNF or NS upon receiving the request through the portal or in reaction to the information supplied by SLA Monitoring module through the NBI. The NFVO shall look at the local catalog, solve the VNF placement problem and interact with the VIM to make an effective deployment of Virtual Machines (VMs) over the hardware substrate. Further the VIM is connected to and SDN controller (e.g. OpenDaylight) to make the chain of VNFs. The VNFM is hence in control of the service life-cycle (migration, rescaling, termination etc).

\subsection{Network Slicing Through Virtualisation}

A fundamental feature of SESAME will be the virtualisation of small cells and their utilisation and partitioning into logically isolated slices, offered to multiple operators/tenants. A hypervisor software is used to create the Network Functions Virtualization Infrastructure (NFVI) on top of the bare hardware where VMs are executed under the control of the SESAME MANO. The main aspect of this innovation will be the capability to accommodate multiple operators within the same infrastructure, satisfying the SLA and requirements of each operator separately. This significantly reduces the costs of the deployed infrastructure (cost of ownership, maintenance, etc), since hosted SCs can be treated as an operating resource instead of a capital expenditure. Under this perspective, the creation of neutral host solutions comes to address also the economic viability of investments done by telecommunications operators.

\subsection{SLA}

The SLA Monitoring module is responsible for gathering information on the use, performance and delivery of network services. It monitors the performance of a tenant network, as well as of the whole SESAME infrastructure. Accordingly, the SLA 
negotiation (encompassing billing issues, accounting and so forth), which lead to liaisons between virtual and physical operators, must be part of an interactive process with the existing support system of the telecommunications operator through appropriate open software. Furthermore, SLA Monitoring will encompass monitoring and analytics as fundamental tools for efficient virtualized network management.

\section{Self-Organising Network Features}

In a multi-tenant scenario like the one considered in SESAME, it should be distinguished between Self-x functions that are tenant-specific (i.e. the configuration of parameters can differ from tenant to tenant) and those that are common to all the tenants. In the following, selected Self-x functions are discussed to illustrate the perspective of tenants and the relation to the SESAME architecture.

\subsection{Self-Planning Functions}

Self-planning is the automation of the decision process to roll out new network nodes in specific areas, identifying adequate configurations and settings of radio parameters, as well as proposing capacity extension for those already deployed (e.g. increasing bandwidth and/or adding new carriers). In SESAME planning of a new cell shall consist of the automatic decision that a new SC has to be deployed in a certain geographical position and the RF planning of such new cell (i.e. transmit power and antenna parameters). The decision is one inherent function to the SCNO, which is responsible to manage the infrastructure to satisfy the capacity demand of different VSCNOs. On the other hand, the spectrum planning function specifies the amount of bandwidth required by a SC (either a new cell or one already deployed), the type of spectrum (e.g. licensed/unlicensed, etc) and carries out the automatic assignment of the spectrum. In SESAME this function can be tenant-specific if slicing of the resources is implemented assigning separate carriers to each tenant.

\subsection{Self-Optimisation Functions}

Once the network is in operational state, self-optimization includes the set of functions to improve or maintain the network performance in terms of coverage, capacity and service quality by tuning the different network settings. In SESAME the Coverage and Capacity Optimization (CCO) function is used to adjust RF parameters based on coverage and capacity targets. This task is accomplished by the SCNO and it cannot be left to a single tenant since these parameters affect all the tenants sharing the same physical SC.

Automatic Neighbor Relations (ANR) is responsible for automatically building the Neighbor Relation Table (NRT) of each small cell. This is fundamental for mobility purposes because handovers can only be executed between neighbor cells. In SESAME, to manage mobility in a multi-tenant scenario, the NRT of each SC has to 
include the relation between the SCs of the SCNO and the cells that belong to a specific tenant.

Mobility Load Balancing (MLB) addresses the problem of uneven traffic distribution in mobile networks. The main target of MLB and traffic steering algorithms is to enable overloaded cells to re-direct part of their traffic to neighbouring less loaded cells, hence alleviating congestion problems. This suits particularly well the deployment proposed by SESAME within the CESC cluster, in which the central decision of the CESCM can provide optimisation of the cluster as a whole. The resulting increased network efficiency using MLB postpones the deployment of additional network capacity, in turn reducing costs. This is usually done through range-expansion [16], achieved by either cell coverage parameter adjustments or mobility parameter adjustments. However, in 4G LTE networks, MLB is known to lead to network performance degradation due to the frequency reuse-1 in this technology [17]. SESAME will develop solutions to remedy this inefficiency.

Admission and congestion control are integral parts of any Quality of Service (QoS) mechanism for networks that support different types of traffic. This is the case in SESAME where a variety of different applications are delivered to the tenants of a CESC. SESAME will utilise admission and congestion control algorithms to deliver in effective manner the SLA agreed between VSCNOs and the SCNO. In traditional networks where the infrastructure is owned by a single tenant, admission and congestion control mechanisms utilise resources (e.g. multi-service packet traffic) that this tenant provides to the end-users. SESAME constitutes a more challenging environment since admission and congestion control mechanisms have to take into account the particular features of the tenants to ensure that services which have specific QoS characteristics can be delivered.

\subsection{Self-Healing Functions}

This is the automation of the processes related to fault management and fault correction, usually associated to hardware and/or software problems, in order to keep the network operational while awaiting a more permanent solution to fix it and/or prevent disruptive problems from arising. In SESAME the concept of cell outage can be extended to both the CESC and CESC cluster. Specifically, CESC outage occurs in case of failure of the small cells, the micro-server or VNFs. Cell outage detection is done first collecting information such as alarms, alters, error messages and key performance indicators at both CESC and CESC cluster levels. Afterward, cell outage detection can be done applying different methods, including data mining (e.g. for large small cells deployment) in order to identify possible misbehaviours [18].

\section{Conclusion}

In this paper we have illustrated the system architecture developed by the 5G-PPP SESAME project, as well features and peculiarities of this system, in the scope of the future $5 \mathrm{G}$ mobile network. We have discussed the essential features of the different modules present in the system and we have clearly highlighted the suitability of 
SESAME to leverage an accrued virtualised network environment. Moreover, we have showed that SESAME incorporates guidelines from standard bodies such as the ETSI NFV ISG. SESAME shall leverage on a cloud-enabled small cell environment at the mobile network edge materialised by the Light DC. Network virtualisation shall allow SESAME to decouple network services from the bare hardware in unprecedented manner, and Self-x features render the system a suitable host for multitenant operators.

\section{Acknowledgments}

The research leading to these results has been supported by the EU funded H2020 5GPPP project SESAME under the grant agreement no 671596.

\section{References}

1. Kim, Y. H., Kim, D. J., Wachter, K.: A Study of Mobile User Engagement (MoEN): Engagement Motivations, Perceived Value, Satisfaction, and Continued Engagement Intention. Decision Support Systems, vol. 56, no. 1, pp. 361--370 (2013)

2. Ericsson Mobility report: On the Pulse of Networked Society (2015)

3. 5G-PPP: https://5g-ppp.eu/

4. "SESAME Project: http://www.sesame-h2020-5g-ppp.eu/

5. Tojib, D., Tsarenko, Y., Sembada, A. Y.: The Facilitating Role of Smart-phones in Increasing Use of Value-Added Mobile Services. New Media \& Society Journal 17: 1220-1240 (2016). First published on February 4, 2014 doi:10.1177/1461444814522951

6. OECD Report: OECD Broadband Statistics Update. http://www.oecd.org/internet/broadband-statistics-update.htm (last accessed April 2016).

7. Deliverable 1.5: Updated Scenarios, Requirements and KPIs for 5G Mobile and Wireless System with Recommendations for Future Investigations. METSI (2015)

8. Andrews, J. G. et al.: What Will 5G Be? IEEE Journal on Selected Areas in Communications, vol. 32, no. 6, pp. 1065--1082 (2014)

9. Kreutz, D., Ramos, F. M., Verissimo, P., Rothenberg, C. E., Azodolmolky, S., Uhlig, S.: Software-Defined Networking: A Comprehensive Survey. Proceedings of the IEEE, vol. 103, no. 1, pp. 14--76 (2014)

10. Chiosi, M., et al.: Network Functions Virtualisation: An Introduction, Benefits, Enablers, Challenges \& Call for Action. SDN and OpenFlow World Congress (2012)

11. ETSI GS NFV 001: Network Functions Virtualization (NFV); Use Cases (2013)

12. Network Functions Virtualisation (NFV); Management and Orchestration. ETSI GS NFVMAN 001 V1.1.1 (2012)

13. Andrews, J. G.: Seven Ways that HetNets are a Cellular Paradigm Shift. IEEE Communications Magazine, vol. 51, no. 3, pp. 136--144 (2013)

14. Osseiran, A. et.al.: Scenarios for 5G Mobile and Wireless Communications: The Vision of the METIS Project. IEEE Communications Magazine, vol. 52, no. 5, pp. 26--35 (2014)

15. Small Cell Virtualization: Functional Splits and Use Cases. SCF 159.06.02 (2016)

16. Ruiz-Avils, J. M., et. Al: Analysis of Limitations of Mobility Load Balancing in a Live LTE System. IEEE Wireless Communications Letters, vol. 4, no. 4 (2015)

17. Mobility Enhancements in HetNets. 3GPP TR 36.839 V11.1.0.

18. Wang, W., Zhang, J., Zhang, Qian.: Cooperative Cell Outage Detection in Self-Organising Femtocell Networks. IEEE INFOCOM, pp. $782--790$ (2013) 